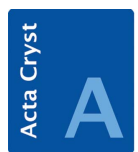

FOUNDATIONS ADVANCES

ISSN 2053-2733

Received 7 April 2020

Accepted 3 June 2020

Edited by A. Altomare, Institute of Crystallography - CNR, Bari, Italy

Keywords: quasicrystals; projection method; inflation rules; diffraction; hyperuniformity.

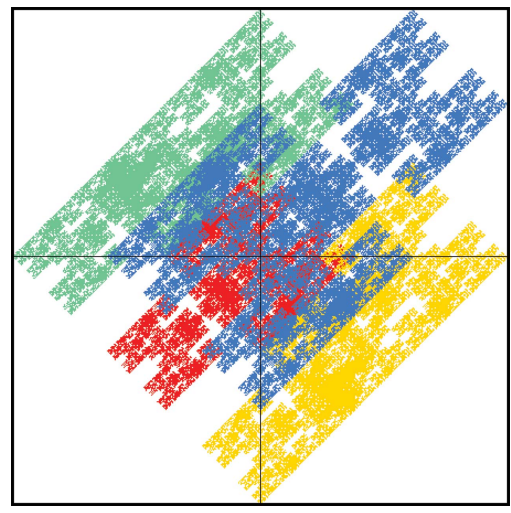

OPEN $\odot$ ACCESS

\section{Inflation versus projection sets in aperiodic systems: the role of the window in averaging and diffraction}

\author{
Michael Baake ${ }^{\mathrm{a}, \mathrm{c}}$ and Uwe Grimm ${ }^{\mathrm{b}, \mathrm{c} *}$ \\ ${ }^{\text {a}}$ Fakultät für Mathematik, Universität Bielefeld, Postfach 100131, 33501 Bielefeld, Germany, ${ }^{\mathbf{b}}$ School of Mathematics and \\ Statistics, The Open University, Walton Hall, Milton Keynes MK7 6AA, UK, and ' School of Natural Sciences, University \\ of Tasmania, Private Bag 37, Hobart TAS 7001, Australia. *Correspondence e-mail: uwe.grimm@open.ac.uk
}

Tilings based on the cut-and-project method are key model systems for the description of aperiodic solids. Typically, quantities of interest in crystallography involve averaging over large patches, and are well defined only in the infinitevolume limit. In particular, this is the case for autocorrelation and diffraction measures. For cut-and-project systems, the averaging can conveniently be transferred to internal space, which means dealing with the corresponding windows. In this topical review, this is illustrated by the example of averaged shelling numbers for the Fibonacci tiling, and the standard approach to the diffraction for this example is recapitulated. Further, recent developments are discussed for cut-and-project structures with an inflation symmetry, which are based on an internal counterpart of the renormalization cocycle. Finally, a brief review is given of the notion of hyperuniformity, which has recently gained popularity, and its application to aperiodic structures.

\section{Introduction}

The discovery of quasicrystals in the early 1980s (Shechtman et al., 1984) not only led to a reconsideration of the fundamental concept of a crystal [see Grimm (2015) and references therein], but also highlighted the need for a mathematically robust treatment of the diffraction of systems that exhibit aperiodic order. The foundations for a rigorous approach were laid by Hof (1995). In particular, the measure-theoretic approach via the autocorrelation and diffraction measures allows for a mathematically rigorous discussion and separation of the different spectral components, the pure point, singular continuous and absolutely continuous parts; see Baake \& Grimm (2012) for background and examples, and ch. 9 in Baake \& Grimm (2013) for a systematic exposition. For general background on the theory of aperiodic order, we refer readers to Pytheas Fogg (2002), Allouche \& Shallit (2003), Queffélec (2010), Baake \& Grimm (2013), Kellendonk et al. (2015), Akiyama \& Arnoux (2020) and references therein.

Within a few years, it was established that regular model sets (Moody, 2000), meaning systems obtained by projection from higher-dimensional lattices via cut-and-project mechanisms with 'nice' windows, have pure point diffraction (Schlottmann, 2000; Richard \& Strungaru, 2017a). We refer readers to the discussion in Baake \& Grimm (2013) for details and examples, and to Baake et al. (2016) for an instructive application of the cut-and-project approach to an experimentally observed structure with 12 -fold symmetry. The result on the pure point nature of diffraction holds for rather general setups, including cut-and-project schemes with non-Euclidean internal spaces. It has recently been generalized to weak model sets of extremal densities (Baake et al., 2017; Richard \& 
Strungaru, 2017b), for which the window may even entirely consist of boundary, that is, has no interior; see also Strungaru $(2017,2020)$ for recent work on pure point spectra.

While systems based on a cut-and-project scheme are generally well understood, this is less so for systems originating from substitution or inflation rules, which constitute another popular method of generating systems with aperiodic order; see Queffélec (2010), Baake \& Grimm (2013), Frettlöh (2017), and references therein for details. There has been recent progress particularly on substitutions of constant length; see Mañibo (2017), Bartlett (2018), Berlinkov \& Solomyak (2019), Baake et al. (2020), Baake, Frank et al. (2019), Bufetov \& Solomyak (2020).

There are familiar examples of inflation-based structures for all spectral types, such as the Fibonacci chain for a pure point diffractive system, the Thue-Morse chain for a system with purely singular continuous diffraction, and the binary Rudin-Shapiro chain as the paradigm of a system with absolutely continuous diffraction; see Pytheas Fogg (2002), Allouche \& Shallit (2003), Baake \& Grimm (2013) for details. When one equips the Rudin-Shapiro chain with balanced weights $( \pm 1)$, it becomes homometric with the binary Bernoulli chain with random weights \pm 1 (Baake \& Grimm, 2009). It is easy to construct inflation-based systems which combine any of these spectral components in their diffraction; see Baake et al. (2013) for examples. As of today, the celebrated Pisot substitution conjecture (which stipulates that an irreducible Pisot substitution has a pure point spectrum) remains open; see Akiyama et al. (2015) for a review of the state of affairs.

While diffraction was the first property to be analysed in detail, many other questions from traditional crystallography and lattice theory require an extension to their aperiodic counterparts (Baake \& Zeiner, 2017). In particular, classic counting problems based on lattices, when reformulated for point sets in aperiodic tilings, need both a conceptual reformulation and new tools to tackle them. The key observation is the necessity to employ averaging concepts, and then tools from dynamical systems and ergodic theory (Queffélec, 2010; Solomyak, 1997; Baake \& Grimm, 2013). If one is in the favourable situation of point sets that emerge from either the projection formalism or an inflation procedure, many of these averaged quantities are well defined and can actually be calculated; see Baake \& Grimm (2003) and references therein. Despite good progress, many questions in this context remain open.

Let us sketch how this introductory review is organized. Our guiding example in this exposition is the classic selfsimilar Fibonacci tiling of the real line. Its descriptions as an inflation set and as a cut-and-project set are reviewed in Section 2. As a simple example of the role of the window in averaging, we discuss the averaged shelling for this system in Section 3. This is followed by a brief review of the standard approach to diffraction in Section 4, where we exploit the description of the Fibonacci point set as a cut-and-project set and the general results for the diffraction of regular model sets.
In Section 5, we recapitulate the recently developed internal cocycle approach. For systems which possess both an inflation and a projection interpretation, such as the Fibonacci tiling, the inflation cocycle can be lifted to internal space. This makes it possible to efficiently compute the diffraction of certain cut-and-project systems with complicated windows, such as windows with fractal boundaries, as are commonly found in inflation structures. To explore this further, we reconsider planar examples, based on the Fibonacci substitution, in Section 6.

Finally, in Section 7, we discuss the use of 'hyperuniformity' as a measure of order in Fibonacci systems. This amounts to an investigation of the asymptotic behaviour of the total diffraction intensity near the origin. It turns out that this can dinstinguish between generic and inflation-invariant choices for the window in the cut-and-project scheme.

\section{The Fibonacci tiling revisited}

Let us start with a paradigm of aperiodic order in one dimension, the classic Fibonacci tiling. It can be defined via the primitive two-letter inflation rule

$$
\varrho: \quad a \rightarrow a b, \quad b \rightarrow a,
$$

where $a$ and $b$ represent tiles (or intervals) of length $\tau=$ $(1+\sqrt{5}) / 2$ and 1 , respectively. The corresponding incidence matrix is given by

$$
M=\left(\begin{array}{ll}
1 & 1 \\
1 & 0
\end{array}\right)
$$

which has the Perron-Frobenius eigenvalue $\tau$. Its left and right eigenvectors read

$$
\langle u|=\frac{\tau+2}{5}(\tau, 1) \quad \text { and } \quad|v\rangle=\left(\tau^{-1}, \tau^{-2}\right)^{\mathrm{T}},
$$

where we employ Dirac's intuitive 'bra-c-ket' notation, which makes it easy to distinguish row and column vectors. We normalize the right eigenvector $|v\rangle$ such that $\langle 1 \mid v\rangle=1$, which means that its entries are the relative frequencies of the tiles. For later convenience, we normalize the left eigenvector $\langle u|$ by setting $\langle u \mid v\rangle=1$, rather than using the vector of natural tile lengths itself. With this normalization, we have

$$
\begin{aligned}
\lim _{n \rightarrow \infty} \tau^{-n} M^{n} & =\frac{\tau+2}{5}\left(\begin{array}{cc}
1 & \tau^{-1} \\
\tau^{-1} & \tau^{-2}
\end{array}\right) \\
& =|v\rangle\langle u|=: P,
\end{aligned}
$$

where $P=P^{2}$ is a symmetric projector of rank 1 with spectrum $\{1,0\}$.

Starting from the legal seed $b \mid a$, where the vertical bar denotes the origin, and iterating the square of the inflation rule $\varrho$ generates a tiling of the real line that is invariant under $\varrho^{2}$; see Example 4.6 in Baake \& Grimm (2013) for details and why it does not matter which of the two fixed points of $\varrho^{2}$ one chooses. Let us use the left endpoints of each interval as control points and denote the set of these points by $\Lambda_{a}$ and $\Lambda_{b}$, respectively. Clearly, since $0 \in \Lambda_{a}$ and all tiles have 
either length $\tau$ or length 1 , all coordinates are integer linear combinations of these two tile lengths, and we have

$$
\Lambda_{a, b} \subset \mathbb{Z}[\tau]=\{m+n \tau: m, n \in \mathbb{Z}\} .
$$

The incidence matrix $M$ only contains information about the number of tiles under inflation, not about their positions. To capture the latter, and thus encode the full information of the inflation, we consider the set-valued displacement matrix

$$
T=\left(\begin{array}{cc}
\{0\} & \{0\} \\
\{\tau\} & \emptyset
\end{array}\right),
$$

where $\emptyset$ denotes the empty set. Note that $T$ is the geometric counterpart of the instruction matrices that are used in the symbolic context (Queffélec, 2010). The matrix elements of $T$ are sets that specify the relative displacement for all tiles under inflation. For instance, the two entries in the first column correspond to a long tile with relative shift 0 and a small tile with shift $\tau$ originating from inflating a long tile. Clearly, the inflation matrix $M$ is recovered if one takes the elementwise cardinality of $T$, noting that the empty set has cardinality 0 .

The inflation rule $\varrho$ induces an iteration on pairs of point sets, namely

$$
\begin{aligned}
& \Lambda_{a}^{(n+1)}=\tau \Lambda_{a}^{(n)} \cup \tau \Lambda_{b}^{(n)}, \\
& \Lambda_{b}^{(n+1)}=\tau \Lambda_{a}^{(n)}+\tau,
\end{aligned}
$$

with suitable initial conditions $\Lambda_{a, b}^{(0)}$. When one starts with the left endpoints of a legal seed, this iteration precisely reproduces the endpoints of the corresponding successive inflation steps. In this case, the union on the right-hand side is disjoint. In particular, for the above choice of $\Lambda_{a, b}$, one needs $\Lambda_{a}^{(0)}=\{0\}$ and $\Lambda_{b}^{(0)}=\{-1\}$.

The point sets $\Lambda_{a, b}$ also have an interpretation as a cut-andproject set. Here, we use the natural (Minkowski) embedding of the module $\mathbb{Z}[\tau]$ in the plane $\mathbb{R}^{2}$, by associating to each $x=m+n \tau \in \mathbb{Z}[\tau]$ its image $x^{\star}=m+n \tau^{\star}=m+n(1-\tau)$ under algebraic conjugation (which maps $\sqrt{5}$ to $-\sqrt{5}$ ). This gives

$$
\begin{aligned}
\mathcal{L} & =\left\{\left(x, x^{\star}\right): x \in \mathbb{Z}[\tau]\right\} \\
& =\left\{\left(m+n \tau, m+n \tau^{\star}\right): m, n \in \mathbb{Z}\right\} \\
& =\left\{m(1,1)+n\left(\tau, \tau^{\star}\right): m, n \in \mathbb{Z}\right\},
\end{aligned}
$$

which is a planar lattice with basis vectors $(1,1)$ and $\left(\tau, \tau^{\star}\right)$; see Baake \& Grimm (2013) and Baake et al. (2016) for details and further examples. Here, we refer to the two one-dimensional subspaces of $\mathbb{R}^{2}=\mathbb{R} \times \mathbb{R}$ as the physical and the internal space, respectively. The physical space hosts our point sets $\Lambda_{a, b}$, while the windows are subsets of the internal space, with the $\star$-map providing the relevant link between the two spaces.

The two point sets $\Lambda_{a, b}$ are given by the projection of all points of $\mathcal{L}$ within two strips; see Fig. 1 . These strips are defined by their cross sections, usually called windows, which are the half-open intervals

$$
W_{a}=[\tau-2, \tau-1) \quad \text { and } \quad W_{b}=[-1, \tau-2) .
$$

With $L=\mathbb{Z}[\tau]$, the projection of $\mathcal{L}$ into physical space, the point sets are thus given by

$$
\Lambda_{a, b}=\left\{x \in L: x^{\star} \in W_{a, b}\right\} .
$$

One of the powerful properties of the cut-and-project approach is that we can switch between the physical space and the internal space, and calculate properties in the latter. Taking the $\star$-image of (5), we obtain the relations

$$
W_{a}=\sigma W_{a} \cup \sigma W_{b}, \quad W_{b}=\sigma W_{a}+\sigma,
$$

where $\sigma=\tau^{\star}=1-\tau$ satisfies $|\sigma|<1$. These relations are an important ingredient for the internal cocycle approach. Due to $|\sigma|<1$, this gives rise to a contractive iterated function system, which has the windows $W_{a, b}$ (or, more precisely, their closures) as its unique solution.

One key property, which can be employed to show that the point sets $\Lambda_{a, b}$ are pure point diffractive, is the fact that the

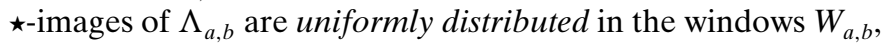
which makes it possible to translate the computation of averaged quantities in physical space to computations in internal space.

\section{Shelling}

Let us discuss a simple example of an averaged quantity, the averaged shelling function for the Fibonacci point set; see Baake \& Grimm (2003) for the concept and various applications to aperiodic systems. The shelling problem is related to the autocorrelation as well as to diffraction; we include it here to demonstrate, in a simple explicit example, the advantages of using internal space for this type of analysis.

For a point set, the shelling problem asks for the number $n(r, x)$ of points that lie on shells of radius $r$, taken with respect to a fixed centre $x$. For an aperiodic point set, this generally depends on the choice of the centre. The averaged shelling numbers $a(r)$ are obtained by taking the average over all choices of centres, where we limit ourselves to centres that are themselves in the point set, so $x \in \Lambda$. Clearly, since we are dealing with a one-dimensional point set, any shell can have at most two points, so $n(r, x) \in\{0,1,2\}$ for all $r \in \mathbb{R}$, with $n(r, x)=0$ if $r \notin \mathbb{Z}[\tau]$, as well as $n(0, x)=a(0)=1$. Clearly,

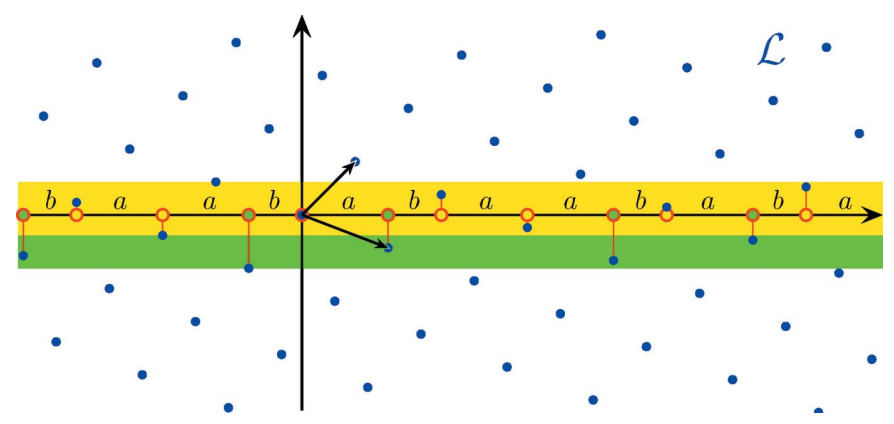

Figure 1

Cut-and-project description of the Fibonacci chain from the lattice $\mathcal{L}$ (blue dots). The windows $W_{a}$ and $W_{b}$ are the cross sections of the yellow and green strips, respectively. 
this also implies that $a(r) \in[0,2]$ for all $r \in \mathbb{R}$, with $a(r)=0$ whenever $r \notin \mathbb{Z}[\tau]$.

Consider a point $x \in \Lambda$ and $r=m+n \tau \in \mathbb{Z}[\tau]$. To compute $n(r, x)$, we have to check whether $x \pm r$ are also in the point set $\Lambda$. From the model set description, we know that $x^{\star} \in W$, and checking whether $x \pm r$ are in $\Lambda$ is equivalent to checking whether $x^{\star} \pm r^{\star} \in W$. In other words, we can express $n(r, x)$ for $r>0$ in terms of the window $W$ as

$$
n(r, x)=1_{W}\left(x^{\star}\right) 1_{W}\left(x^{\star}+r^{\star}\right)+1_{W}\left(x^{\star}\right) 1_{W}\left(x^{\star}-r^{\star}\right),
$$

where $1_{W}$ denotes the indicator (or characteristic) function of the window $W$, defined by

$$
1_{W}(x)= \begin{cases}1 & \text { if } x \in W \\ 0 & \text { otherwise }\end{cases}
$$

While it is possible to perform this computation for any given value of $x$ and $r$, there is no simple closed formula for these coefficients.

To obtain the averaged shelling number, we have to consider all $x \in \Lambda$ as centres, each with the same weight, which means averaging over all $x^{\star} \in W$. Define $\nu(r)=v(-r)$ as the relative frequency to find one point of $\Lambda$ at $x$ as well as one at $x+r$, so $a(0)=v(0)=1$ and $a(r)=2 v(r)$ for $r>0$, to account for the points on both sides. Now, for $r \in \mathbb{Z}[\tau]$, the frequency $v(r)$ of having both $x^{\star} \in W$ and $x^{\star}+r^{\star} \in W$ can be calculated as the overlap length between the window $W$ and the shifted window $W-r^{\star}$, divided by the length of $W$, which is $|W|=\tau$. This is correct because the uniform distribution of points in the window (Moody, 2002; Baake \& Grimm, 2013) implies that the frequency of any configuration is proportional to the length of the corresponding sub-window. Clearly, the length of the overlap between these two intervals is 0 whenever $\left|r^{\star}\right|>\tau$, and otherwise decreases linearly with $\left|r^{\star}\right|$, so we get

$$
\begin{aligned}
v(r) & =\frac{\left|W \cap\left(W-r^{\star}\right)\right|}{|W|} \\
& = \begin{cases}1-\frac{||^{\star} \mid}{\tau} & \text { if } r \in \mathbb{Z}[\tau] \text { and }\left|r^{\star}\right| \leq \tau, \\
0 & \text { otherwise. }\end{cases}
\end{aligned}
$$

Consequently, the averaged shelling numbers for the Fibonacci point set are given by

$$
a(r)= \begin{cases}1 & \text { if } r=0, \\ 2\left(1-\frac{\left|r^{\star}\right|}{\tau}\right) & \text { if } r \in \mathbb{Z}[\tau] \text { with }\left|r^{\star}\right| \leq \tau, \\ 0 & \text { otherwise. }\end{cases}
$$

Note that $a(r)$, for $r \in \mathbb{Z}[\tau]$, is a simple function of $r^{\star}$, but that it behaves rather erratically if one looks at it as a function of $r$; see Fig. 2. The reason behind this observation is the total discontinuity of the $\star$-map from physical to internal space.

For the one-dimensional example at hand, the numbers $v(r)$ are nothing but the relative probabilities of finding two points at a distance $r$, and thus the (relatively normalized) autocorrelation coefficients of the point set $\Lambda$. As such, they are intimately connected to the diffraction of this point set. Clearly, correlations are much easier to handle in internal space, where we can calculate them via volumes of intersections of windows, as we shall see shortly.

\section{Standard approach to diffraction}

Here, we start with a brief summary of the derivation of the diffraction spectrum for the Fibonacci point set $\Lambda=\Lambda_{a} \cup \Lambda_{b}$, considered as a cut-and-project set $\Lambda=\left\{x \in L: x^{\star} \in W\right\}$ with $W=W_{a} \cup W_{b}$. Assume that we place point scatterers of unit scattering strength at all points $x \in \Lambda$, and consider the corresponding Dirac comb

$$
\omega=\delta_{\Lambda}:=\sum_{x \in \Lambda} \delta_{x} .
$$

We associate to $\omega$ the autocorrelation $\gamma=\omega \circledast \widetilde{\omega}$, where $\widetilde{\omega}=$ $\delta_{-\Lambda}$ is the 'flipped-over' (reflected) version of $\omega$ and $\circledast$ denotes volume-averaged (or Eberlein) convolution (Baake \& Grimm, 2013, Section 8.8). The diffraction measure $\widehat{\gamma}$ is the Fourier transform of the autocorrelation.

From the general diffraction theory for cut-and-project sets with well-behaved windows, we know that the diffraction measure of this system is a pure point measure, so consists of Bragg peaks only. These Bragg peaks are located on the projection of the entire dual lattice

$$
\mathcal{L}^{*}=\frac{1}{\sqrt{5}}\{m(\tau-1, \tau)+n(1,-1): m, n \in \mathbb{Z}\}
$$

to the physical space (the first coordinate), which is $L^{\circledast}=$ $(1 / \sqrt{5}) \mathbb{Z}[\tau]$. We call this set the Fourier module of the Fibonacci point set; it coincides with the dynamical spectrum (in additive notation) in the mathematical literature. Note that $1 / \sqrt{5}=(2 \tau-1) / 5$, hence $L^{\circledast} \subset \mathbb{Q}(\tau)$, which means that the $\star$-map is well defined for all $k \in L^{\circledast}$. The Fourier module is a dense subset of $\mathbb{R}$, which means that the diffraction consists of Bragg peaks on a dense set in space, where the intensities are locally summable.

The diffraction measure is thus the countable sum

$$
\widehat{\gamma}=\sum_{k \in L^{\circledast}}|A(k)|^{2} \delta_{k}
$$

where the diffraction amplitudes, or Fourier-Bohr (FB) coefficients, are given by the general formula

$$
A(k)=\frac{\operatorname{dens}(\Lambda)}{\operatorname{vol}(W)} \widehat{1_{W}}\left(-k^{\star}\right)=\frac{\operatorname{dens}(\Lambda)}{\operatorname{vol}(W)} \widehat{1_{W}}\left(k^{\star}\right)
$$

for all $k \in L^{\circledast}$, and vanish otherwise. Here,
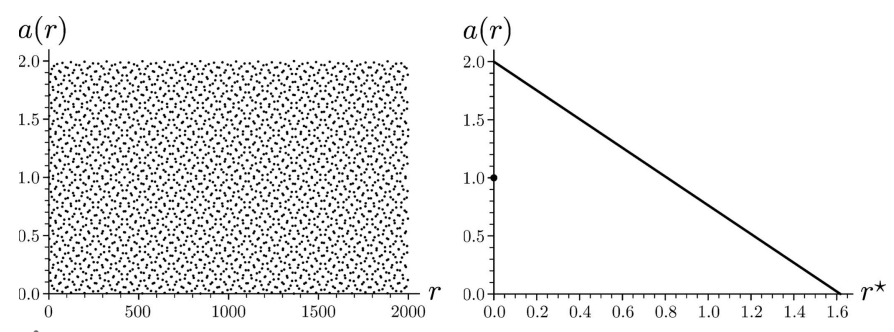

Figure 2

Averaged shelling numbers $a(r)$ for the Fibonacci point set as a function of $r$ (left) and $r^{\star}$ (right). 


$$
\begin{aligned}
& \widehat{g}(k)=\int_{-\infty}^{\infty} \exp (-2 \pi \mathrm{i} k x) g(x) \mathrm{d} x \text { and } \\
& \overline{\widehat{g}}(k)=\int_{-\infty}^{\infty} \exp (2 \pi \mathrm{i} k x) g(x) \mathrm{d} x
\end{aligned}
$$

denote the Fourier and the inverse Fourier transform of a real-valued $L^{1}$-function $g$. With $\operatorname{dens}(\Lambda)=(\tau+2) / 5$ and $\operatorname{vol}(W)=|W|=\tau$, equation (9) evaluates to

$$
\begin{aligned}
A(k) & =\frac{1}{\sqrt{5}} \int_{-1}^{\tau-1} \exp \left(2 \pi \mathrm{i} k^{\star} y\right) \mathrm{d} y \\
& =\frac{\tau}{\sqrt{5}} \exp \left[\pi \mathrm{i} k^{\star}(\tau-2)\right] \operatorname{sinc}\left(\pi \tau k^{\star}\right)
\end{aligned}
$$

where $\operatorname{sinc}(x)=\sin (x) / x$. Hence, the diffraction intensities are

$$
I(k)=|A(k)|^{2}=\left[\frac{\tau}{\sqrt{5}} \operatorname{sinc}\left(\pi \tau k^{\star}\right)\right]^{2}
$$

for all $k \in L^{\circledast}$, and 0 otherwise. This is illustrated in Fig. 3 . Note that $I(k)$ can vanish for some $k \in L^{\circledast}$, in which case we talk of an extinction of the Bragg peak. For the Fibonacci system, this may happen for specific choices of the scattering strengths (such as in our simple case, where we chose them to be 1 for all points in $\Lambda$ ). However, for a generic choice of weights [see (11) below], there will be no extinctions, and we will have a Bragg peak for all $k \in L^{\circledast}$.

The corresponding autocorrelation measure $\gamma$ can be expressed in terms of the (dimensionless) pair correlation coefficients

$$
v(r):=\frac{\operatorname{dens}[\Lambda \cap(\Lambda-r)]}{\operatorname{dens}(\Lambda)}=v(-r),
$$

which are positive for all $r \in \Lambda-\Lambda \subset \mathbb{Z}[\tau]$ and vanish for all other distances $r$. These are precisely the coefficients we defined in equation (8) to compute the shelling numbers. The link between the two expressions is provided by the $\star$-map and the uniform distribution of $\Lambda^{\star}$ in the window $W$. In terms of these pair correlation coefficients, the autocorrelation measure is

$$
\gamma=\operatorname{dens}(\Lambda) \sum_{r \in \Lambda-\Lambda} \nu(r) \delta_{r},
$$

which is a pure point measure supported on the difference set $\Lambda-\Lambda$.

More generally, we may associate two different, in general complex, scattering strengths $u_{a}$ and $u_{b}$ to the points in $\Lambda_{a}$ and $\Lambda_{b}$, respectively, and consider the weighted Dirac comb $\omega=u_{a} \delta_{\Lambda_{a}}+u_{b} \delta_{\Lambda_{b}}$. In this case, the diffraction intensity for all wavenumbers $k \in L^{\circledast}$ is given by the superposition

$$
I(k)=\left|u_{a} A_{a}(k)+u_{b} A_{b}(k)\right|^{2}
$$

of the corresponding FB amplitudes

$$
\begin{aligned}
A_{a, b}(k) & =\frac{\operatorname{dens}\left(\Lambda_{a, b}\right)}{\operatorname{vol}\left(W_{a, b}\right)} \widehat{1_{W_{a, b}}}\left(-k^{\star}\right) \\
& =\frac{\operatorname{dens}(\Lambda)}{\operatorname{vol}(W)} \widehat{1_{W_{a, b}}}\left(-k^{\star}\right)=\frac{1}{\sqrt{5}} \widehat{1_{W_{a, b}}}\left(-k^{\star}\right) .
\end{aligned}
$$

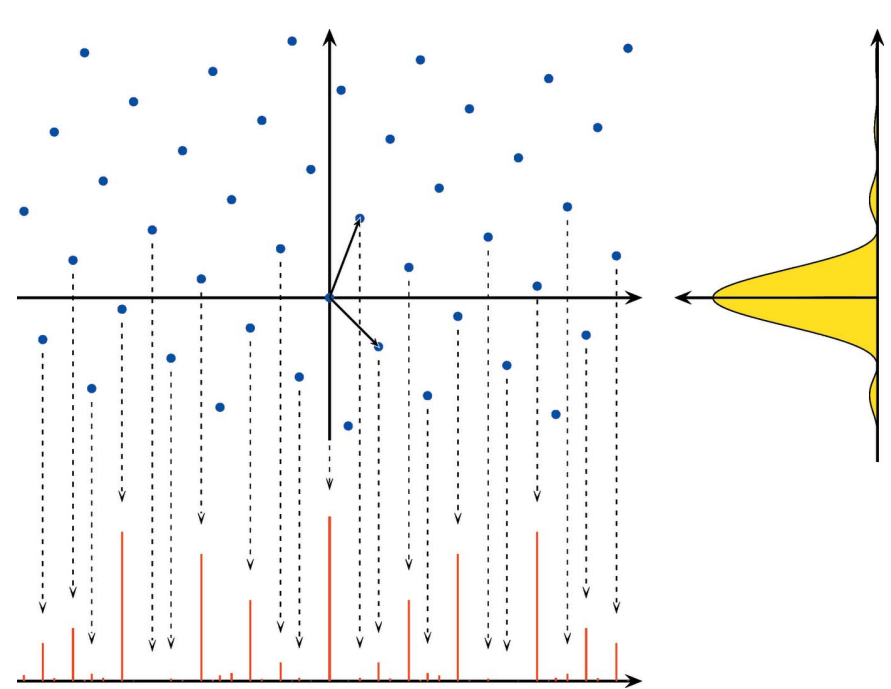

Figure 3

Schematic construction of the diffraction measure of the Fibonacci point set from the dual lattice $\mathcal{L}^{*}$ (blue dots). A point $\left(k, k^{\star}\right) \in \mathcal{L}^{*}$ results in a Bragg peak at $k \in L^{\circledast}$ of intensity given by the value of the function on the right-hand side evaluated at $k^{\star}$. Note that some Bragg peaks may be extinct, if the intensity function vanishes at $k^{\star}$.

The corresponding autocorrelation measure can once more be expressed in terms of pair correlation functions, now distinguishing points in $\Lambda_{a}$ and $\Lambda_{b}$,

$$
v_{\alpha \beta}(r)=\frac{\operatorname{dens}\left[\Lambda_{\alpha} \cap\left(\Lambda_{\beta}-r\right)\right]}{\operatorname{dens}(\Lambda)}=v_{\beta \alpha}(-r) .
$$

These coefficients are positive for all $r \in \Lambda_{\beta}-\Lambda_{\alpha}$ and vanish otherwise, and in particular satisfy the relation

$$
\sum_{\alpha, \beta \in\{a, b\}} v_{\alpha \beta}(r)=v(r) .
$$

The relation (9) between the FB coefficients and the Fourier transform of the compact windows holds for any regular model set, which is a cut-and-project set with some 'niceness' constraint on the window; see Theorem 9.4 in Baake \& Grimm (2013) for details. While this works well for many of the nice examples with polygonal windows, it becomes practically impossible to compute the FB coefficients in this way if the windows are compact sets with fractal boundaries. Such windows naturally arise for cut-and-project sets which also possess an inflation symmetry. Indeed, some of the structure models of icosahedral quasicrystals, see Takakura et al. (2007) for an example, feature experimentally determined windows whose shapes may indicate first steps of a fractal construction of the boundary.

Let us therefore explain a different approach that will permit an efficient computation of the diffraction also for such, more complicated, situations.

\section{Renormalization and internal cocycle}

Let us reconsider our motivating example, the Fibonacci point sets $\Lambda_{a, b}$ of equation (6). We will use both their inflation structure and their description as cut-and-project sets. Here, 
we make use of the iteration (5) and the corresponding relation (7) for the windows (or, more precisely, the closure of the windows). This inflation structure induces the following relation between the characteristic functions of the windows,

$$
1_{W_{a}}=1_{\sigma W_{a} \cup \sigma W_{b}} \text { and } 1_{W_{b}}=1_{\sigma W_{a}+\sigma},
$$

where we again set $\sigma=\tau^{\star}=1-\tau$. Since the (closed) windows only share at most boundary points, we observe that $1_{\sigma W_{a} \cup \sigma W_{b}}$ $=1_{\sigma W_{a}}+1_{\sigma W_{b}}$ holds as an equality of $L^{1}$-functions. Now, we can apply the Fourier transform, which yields the relations

$$
\widehat{1_{W_{a}}}=\widehat{1_{\sigma W_{a}}}+\widehat{1_{\sigma W_{b}}} \text { and } \widehat{1_{W_{b}}}=\widehat{1_{\sigma W_{a}+\sigma}} .
$$

These equations capture the action of the inflation in internal space in terms of functional equations for the Fourier transform of the windows, which in turn determine the diffraction. In what follows, it turns out to be more convenient to work with the inverse Fourier transform. Note that, by an elementary change of variable calculation in the Fourier integral, one has

$$
\widehat{\widehat{1_{\alpha K+\beta}}}(y)=|\alpha| \exp (2 \pi \mathrm{i} \beta y) \widehat{\widehat{1_{K}}}(\alpha y)
$$

for arbitrary $\alpha, \beta \in \mathbb{R}$ with $\alpha \neq 0$ and any compact set $K \subset \mathbb{R}$. This can be used to express the functions in (13) with $\sigma$-scaled and shifted windows in terms of the indicator functions of the original windows.

Indeed, defining

$$
h_{a, b}:=\widehat{\widehat{1_{a, b}}}
$$

for the two functions involving the original windows, and using equation (14), we can rewrite equation (13) as

$$
\left(\begin{array}{l}
h_{a} \\
h_{b}
\end{array}\right)(y)=|\sigma| \underline{B}(y)\left(\begin{array}{l}
h_{a} \\
h_{b}
\end{array}\right)(\sigma y)
$$

with the matrix

$$
\underline{B}(y):=\left[\begin{array}{cc}
1 & 1 \\
\exp (2 \pi \mathrm{i} \sigma y) & 0
\end{array}\right] .
$$

The matrix $\underline{B}$ is obtained by first taking the $\star$-map of the setvalued displacement matrix $T$ of equation (4) and then its inverse Fourier transform. For this reason, $\underline{B}$ is called the internal Fourier matrix (Baake \& Grimm, 2019b), to distinguish it from the Fourier matrix of the renormalization approach in physical space (Baake \& Gähler, 2016; Baake, Frank et al., 2019); see Bufetov \& Solomyak $(2018,2020)$ for various extensions with more flexibility in the choice of the interval lengths.

In Dirac notation, we set $|h\rangle=\left(h_{a}, h_{b}\right)^{\mathrm{T}}$, which satisfies $|h(0)\rangle=\tau|v\rangle$ with the right eigenvector $|v\rangle$ of the substitution matrix $M$ from equation (2). Applying the iteration (16) $n$ times then gives

$$
|h(y)\rangle=|\sigma|^{n} \underline{B}^{(n)}(y)\left|h\left(\sigma^{n} y\right)\right\rangle
$$

where

$$
\underline{B}^{(n)}(y)=\underline{B}(y) \underline{B}(\sigma y) \ldots \underline{B}\left(\sigma^{n-1} y\right) .
$$

In particular, these matrices satisfy $\underline{B}^{(1)}=\underline{B}$ and $\underline{B}^{(n)}(0)=M^{n}$ for all $n \in \mathbb{N}$, where $M$ is the substitution matrix from equation (1), as well as the relations

$$
\underline{B}^{(n+m)}(y)=\underline{B}^{(n)}(y) \underline{B}^{(m)}\left(\sigma^{n} y\right)
$$

for any $m, n \in \mathbb{N}$. Note that $\underline{B}^{(n)}(y)$ defines a matrix cocycle, called the internal cocycle, which is related to the usual inflation cocycle (in physical space) by an application of the $\star$-map to the displacement matrices of the powers of the inflation rule; compare Baake, Gähler \& Mañibo (2019), Baake \& Grimm $(2019 b)$, and see Bufetov \& Solomyak $(2018,2020)$ for a similar approach. Note also that $|\sigma|<1$, which means that $\sigma^{n}$ approaches 0 exponentially fast as $n \rightarrow \infty$. We can exploit this exponential convergence to efficiently compute the diffraction amplitudes, which are proportional to the elements of the vector $|h\rangle$.

Considering the limit as $n \rightarrow \infty$ in equation (18), one can show that

$$
|h(y)\rangle=C(y)|h(0)\rangle
$$

with

$$
C(y)=\lim _{n \rightarrow \infty}|\sigma|^{n} \underline{B}^{(n)}(y),
$$

which exists pointwise for every $y \in \mathbb{R}$. In fact, one has compact convergence, which implies that $C(y)$ is continuous (Baake \& Grimm, 2019b, Theorem 4.6 and Corollary 4.7). Clearly, since $\underline{B}^{(n)}(0)=M^{n}$, we have $C(0)=P$ with the projector $P=|v\rangle\langle u|$ from equation (3).

Using equation (19) with $m=1$ and letting $n \rightarrow \infty$, one obtains

$$
\tau C(y)=C(y) M,
$$

since $|\sigma|=\tau^{-1}$. This relation implies that each row of $C(y)$ is a multiple of the left eigenvector $\langle u|$ of the substitution matrix $M$ from equation (2), so there is a vector-valued function $|c(y)\rangle$ such that

$$
C(y)=|c(y)\rangle\langle u|
$$

holds with $|c(y)\rangle=\left[c_{a}(y), c_{b}(y)\right]^{\mathrm{T}}$, where we have $|c(0)\rangle=|v\rangle$.

From equations (20) and (22), we obtain

$$
|h(y)\rangle=|c(y)\rangle\langle u \mid h(0)\rangle=\tau|c(y)\rangle,
$$

and the inverse Fourier transforms of the windows from equation (15) are thus encoded in the matrix $C$.

For the Fibonacci case, we can calculate $|c(y)\rangle$ by taking the Fourier transforms of the known windows $W_{a, b}$ to obtain

$$
c_{a}(y)=\frac{\exp [2 \pi \mathrm{i}(\tau-1) y]-\exp [2 \pi \mathrm{i}(\tau-2) y]}{2 \pi \mathrm{i} y}
$$

and

$$
c_{b}(y)=\frac{\exp [2 \pi \mathrm{i}(\tau-2) y]-\exp (-2 \pi \mathrm{i} y)}{2 \pi \mathrm{i} y} .
$$

Note that these functions never vanish simultaneously, so $C(y)$ is always a matrix of rank 1 . However, taking the Fourier transform of the windows takes us essentially back to the standard approach. 
The main benefit of the internal cocycle approach is that it applies in other situations, where no explicit calculation of the (inverse) Fourier transform of the windows is feasible. This is achieved via approximating $C(y)$ by $|\sigma|^{n} \underline{B}^{(n)}(y)$ for a sufficiently large $n$, such that $|\sigma|^{n} y$ is small and $C(y)$ is approximated sufficiently well. This works because the (closed) windows are compact sets, so that their (inverse) Fourier transforms are continuous functions. The convergence of this approximation is exponentially fast. We refer readers to Baake \& Grimm (2019b) for further details and an extension of the cocycle approach to more general inflation systems, and to Baake \& Grimm (2020) for a planar example.

From the general formula (9) for regular model sets, the FB amplitudes are

$$
A_{\Lambda_{a, b}}(k)=\frac{h_{a, b}\left(k^{\star}\right)}{\sqrt{5}}=\frac{\tau}{\sqrt{5}} c_{a, b}\left(k^{\star}\right)
$$

for $k \in L^{\circledast}$. So, the relevant input is the knowledge of the Fourier module, which determines where the Bragg peaks are located. Then, one can approximate $C$ by evaluating the matrix product in equation (21), for any chosen $k \in L^{\circledast}$, at $y=k^{\star}$ and with a sufficiently large $n$. In what follows, numerical calculations and illustrations are based on this cocycle approach due to its superior speed and accuracy in the presence of complex windows.

\section{Fractally bounded windows}

The internal cocycle approach of Section 5 was first applied to a ternary inflation tiling with the smallest PisotVijayaraghavan (PV) number (also known as the 'plastic number') as its inflation multiplier (Baake \& Grimm, 2020). In the cut-and-project description, the internal space of this onedimensional tiling is two-dimensional, and the windows are Rauzy fractals (Pytheas Fogg, 2002). This means that the windows are still topologically regular, so each window is the closure of its interior, but they have a fractal boundary of zero Lebesgue measure. Consequently, the general diffraction result for model sets still applies, and the diffraction is given by the Fourier transform of the windows as described above. In turn, this means that the internal cocycle approach applies and can be used to compute the Fourier transforms and the

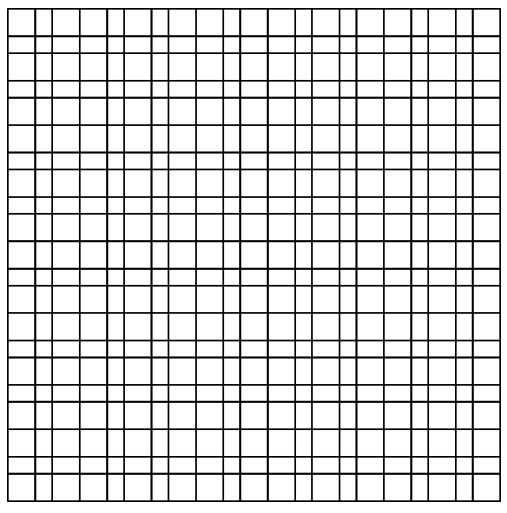

Figure 4

Patch of the square Fibonacci tiling. diffraction intensities for such tilings; see Baake \& Grimm (2020) for details.

Here, we discuss examples of planar projection tilings with fractally bounded windows, which are based on direct product variations (DPVs) (Sadun, 2008; Frank, 2015) of Fibonacci systems, as recently described by Baake et al. (2021). Clearly, if one considers a direct product structure based on the Fibonacci tiling, one obtains a tiling of the plane, called the square Fibonacci tiling. This tiling has been used as a toy model for the study of electronic properties (Lifshitz, 2002; Even-Dar Mandel \& Lifshitz, 2008; Damanik \& Gorodetski, 2018), but has been observed experimentally to form in a molecular overlayer on a twofold surface of an icosahedreal quasicrystal (Coates et al., 2018). It is built from four prototiles, a large square of edge length $\tau$, a small square of edge length 1 , and two rectangles with a long $(\tau)$ and a short (1) edge; see Fig. 4.

As a direct product of inflation tilings, this two-dimensional square Fibonacci tiling also possesses an inflation rule, which takes the form

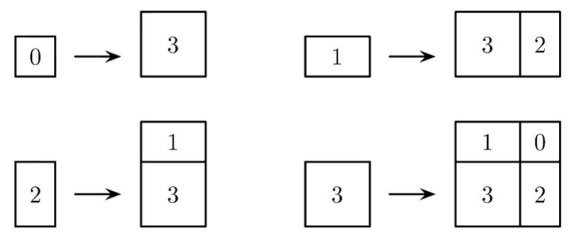

where we labelled the small and large squares by 0 and 3 , and the two rectangles by 1 and 2, respectively. A DPV is now obtained by modifying these rules while keeping the stone inflation character intact, thus probing the ideas of Clark \& Sadun (2006) into a slightly different direction. Clearly, there are two possibilities to rearrange the images of the rectangles by swapping the two tiles, and a close inspection shows that there are altogether 12 ways of rearranging the image of the large square. This means that there are 48 distinct inflation rules in total, which all share these prototiles and the same inflation matrix.

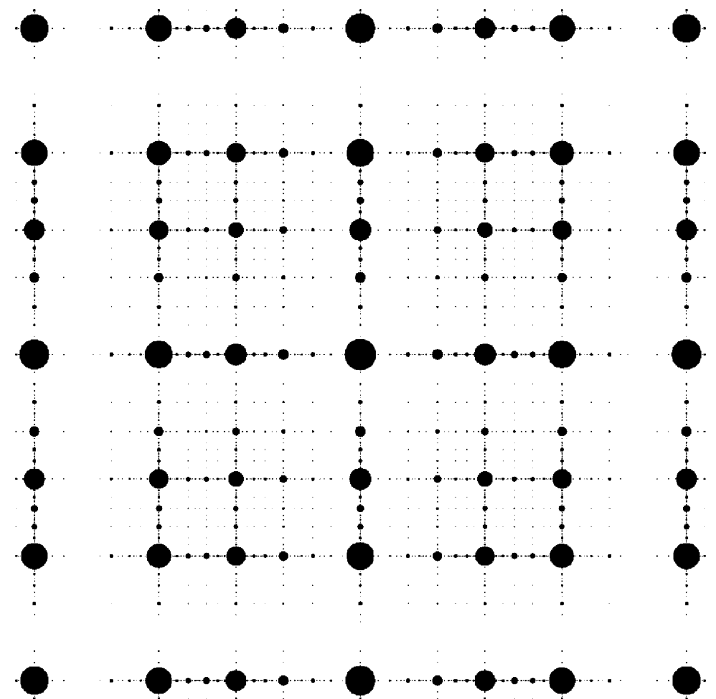

Figure 5

Central part of the diffraction image of the square Fibonacci tiling. 
Due to the direct product structure, the square Fibonacci tiling clearly possesses a cut-and-project description. The windows for the four prototiles are obtained as products of the original windows. The product structure thus extends to the diffraction measure, which is supported on the Fourier module

$$
L^{\circledast} \times L^{\circledast},
$$

where $L^{\circledast}=(1 / \sqrt{5}) \mathbb{Z}[\tau]$ is the Fourier module of the onedimensional Fibonacci tiling. The diffraction amplitudes are also given by products of those for the one-dimensional system, and are thus easy to compute. An illustration of the diffraction pattern is shown in Fig. 5. Here, Bragg peaks are represented by discs, centred at the position of the peak, with areas proportional to their intensities.

It turns out that all $48 \mathrm{DPV}$ inflation tilings are regular model sets, and hence are pure point diffractive; see Theorem 5.2 in Baake et al. (2021). They all share the same Fourier module, $L^{\circledast} \times L^{\circledast}$. This implies that the Bragg peaks are always located at the same positions (where we disregard possible extinctions). However, their intensities are determined by the Fourier transform of the windows, and it turns out that the windows of these DPVs can differ substantially.

In particular, 20 of these DPVs possess windows of Rauzy fractal type, of which there are three different types, called 'castle', 'cross' and 'island' by Baake et al. (2021). They have different fractal dimension of the window boundaries, which are approximately $1.875,1.756$ and 1.561 , respectively. As the dimensions are all smaller than two, is it obvious that these boundaries have zero Lebesgue measure.

In what follows, we are going to illustrate some properties of these DPVs with three examples, one for each of these fractally bounded window types. The inflation rules for the three examples have the same images for the small square (tile 0 ) and both rectangles (tiles 1 and 2) as the square Fibonacci

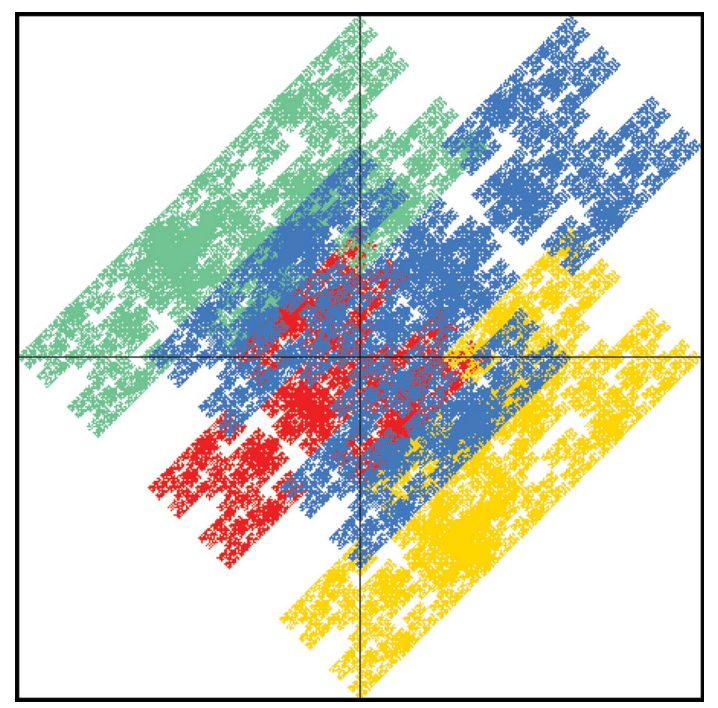

Figure 6

Castle-type window for the DPV (25). The windows for the four types of tiles are distinguished by colour, namely red (0), yellow (1), green (2) and blue (3). The outer box marks the square $[-\tau, \tau]^{2}$, with the coordinate axes indicated as well. rule of equation (24), and thus only differ in the image of the large square (tile 3 ). For a discussion of the complete set of 48 DPVs, we refer readers to Baake et al. (2021).

For the castle-type windows of Fig. 6, we use the inflation

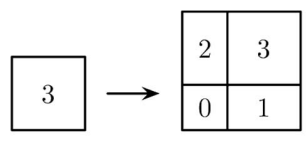

for the large square. Note that this rule dissects the inflated large square such that there is a reflection symmetry along the main diagonal, which will be reflected in a symmetry of the tiling (which maps the squares onto themselves and interchanges the rectangles). This is also apparent for the windows in Fig. 6. The windows for the large and small squares are

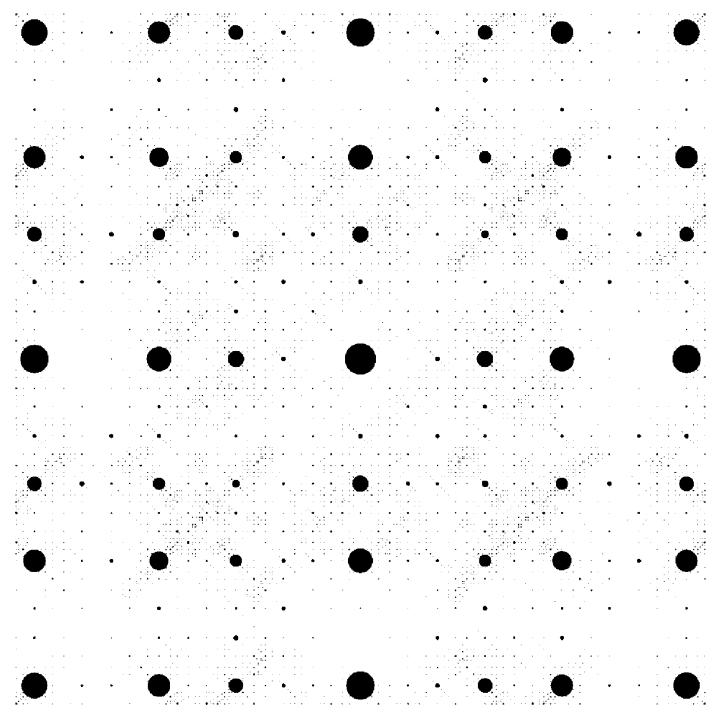

Figure 7

Diffraction image of the DPV (25).

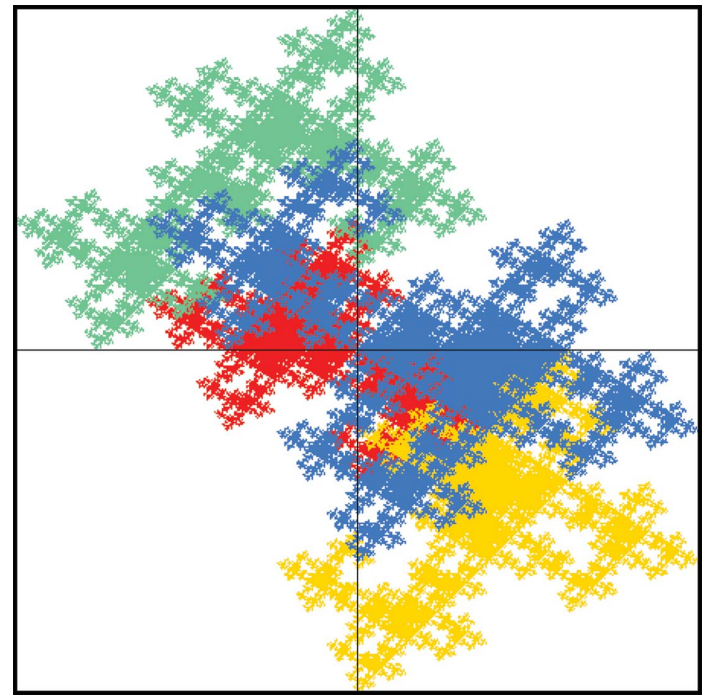

Figure 8

Cross-type window for the DPV (26). 
mapped onto themselves under reflection at the main diagonal, while the windows for the rectangular tiles are interchanged. The diffraction pattern also respects this symmetry; see Fig. 7.

For the cross-type windows, the inflation of the large square is given by

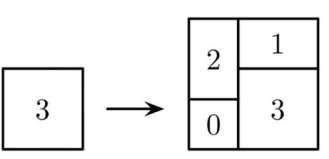

which, in contrast to the previous example, has no reflection symmetry. Consequently, neither the windows shown in Fig. 8 nor the diffraction image illustrated in Fig. 9 have any reflection symmetry.

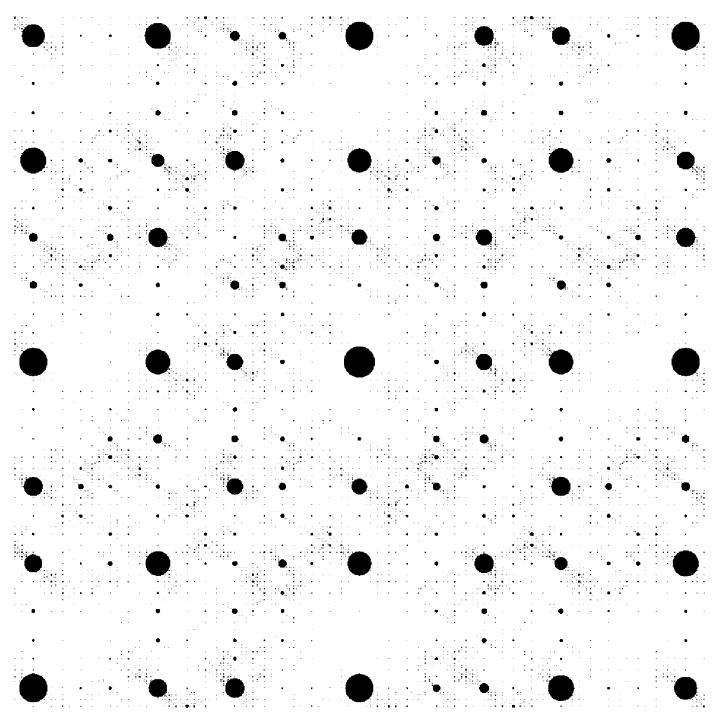

Figure 9

Diffraction image of the DPV (26).

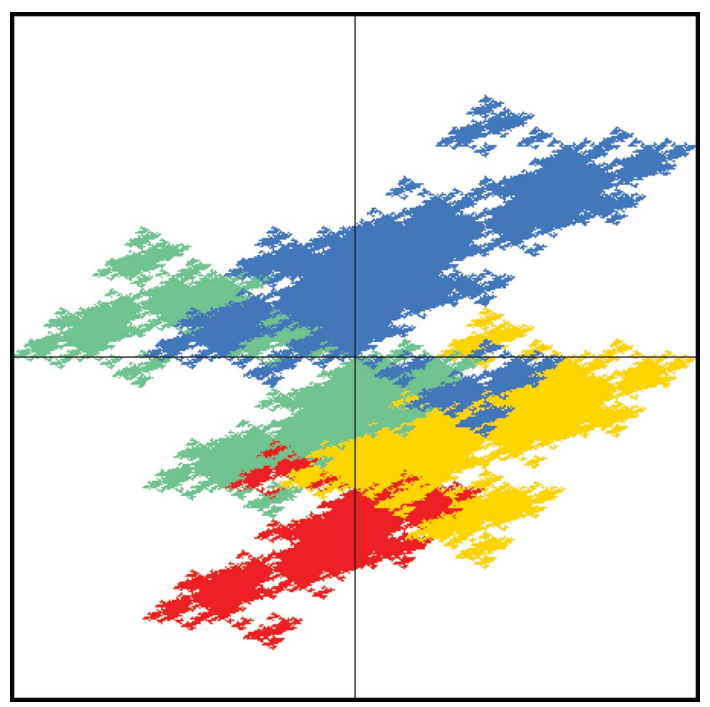

Figure 10

Island-type window for the DPV (27).
The same is true for the final example with the island-type window shown in Fig. 10. This corresponds to the inflation

$$
\begin{array}{|l|l|l|}
\hline \multirow{2}{*}{3} & \multicolumn{1}{|c|}{3} \\
\hline 2 & 3 \\
\cline { 1 - 2 } & 1 \\
\hline
\end{array}
$$

of the large square tile. The corresponding diffraction pattern is illustrated in Fig. 11.

Comparing the diffraction patterns of Figs. 7, 9 and 11 with those of the square Fibonacci tiling shown in Fig. 5, we note that the strongest peaks are almost unchanged, while the intensities of the weaker peaks show some intriguing behaviour. The reason for this behaviour is that all three model sets are subsets of a common Meyer set, and the so-called $\varepsilon$-dual characters of the difference set of this Meyer set, for small $\varepsilon$, always give rise to high-intensity Bragg peaks; see Strungaru (2013) for details. This is the reason why the strongest peaks stay almost the same.

For the fractally bounded windows, one generally sees more peaks, which is due to the larger spread of the window in internal space, and the slower asymptotic decay of the Fourier transform of the window (as $k^{\star} \rightarrow \infty$ ). With limited resolution, some of the intensity distributions on these peaks could resemble continuous components, so might potentially be mistaken as such in experiments.

\section{Diffraction and hyperuniformity}

The discovery of quasicrystals highlighted the lack of a clear definition of the concept of order. In crystallography, diffraction is the main tool to detect long-range order, and a pure point diffraction is generally associated with an ordered, (quasi)crystalline structure, while absolutely continuous diffraction is typically seen as an indication of random disorder [but see Frank (2003), Baake \& Grimm (2009), Chan

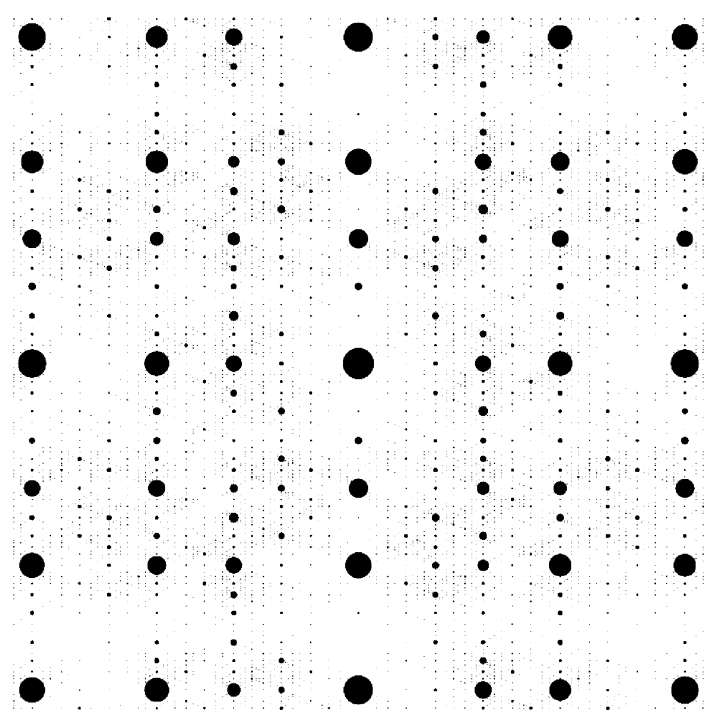

Figure 11

Diffraction image of the DPV (27). 
\& Grimm (2017), Chan et al. (2018) for examples of deterministic structures that show absolutely continuous diffraction]. Here, we briefly discuss a related concept that has recently gained popularity.

From the original idea of using the degree of '(hyper)uniformity' in density fluctuations in many-particle systems (Torquato \& Stillinger, 2003; Brauchart et al., 2019, 2018) to characterize their order, the scaling behaviour of the total diffraction intensity near the origin has emerged as a possible measure to capture long-distance correlations. As far as aperiodic structures are concerned, there are in fact a number of early, partly heuristic, results in the literature (Luck, 1993; Aubry et al., 1988; Godrèche \& Luck, 1990). These have recently been reformulated and extended (Oğuz et al., 2017, 2019) and rigorously established (Baake \& Grimm, 2019a), using exact renormalization relations for primitive inflation rules (Baake, Frank et al., 2019; Baake \& Gähler, 2016; Mañibo, 2017, 2019; Baake, Gähler \& Mañibo, 2019; Baake et al., 2018); see also Fuchs et al. (2019) for results for some planar aperiodic tilings.

For the investigation of scaling properties, we follow the existing literature and define

$$
Z(k):=\widehat{\gamma}((0, k]),
$$

which is a modified version of the distribution function of the diffraction measure. Here, $Z(k)$ is the total diffraction intensity in the half-open interval $(0, k]$, and thus ignores the central peak. Due to the point reflection symmetry of $\widehat{\gamma}$ with respect to the origin, this quantity can also be expressed as

$$
Z(k)=\frac{1}{2}(\widehat{\gamma}([-k, k])-\widehat{\gamma}(\{0\})) .
$$

The interest in the scaling of $Z(k)$ as $k \rightarrow 0$ is motivated by the intuition that the small- $k$ behaviour of the diffraction measure probes the long-wavelength fluctuations in the structure. As the latter is related to the variance in the distribution of patches, it can serve as an indicator for the degree of uniformity of the structure (Torquato \& Stillinger, 2003). It is obvious that any periodic structure leads to $Z(k)=0$ for all sufficiently small wavenumbers $k$.

Here, we review the result for variants of the onedimensional Fibonacci model sets considered above, where we now allow for changes of the windows. For a general discussion of this approach and more examples of systems with different types of diffraction, we refer readers to Baake \& Grimm (2019a) and references therein.

Let us look at the diffraction for a cut-and-project set with the same setup as the Fibonacci tiling considered in Section 4, but with the window $W$ replaced by an arbitrary finite interval of length $s$. Note that these tilings, in general, do not possess an inflation symmetry. Nevertheless, the diffraction intensity is still of the form (10), but now featuring the interval length $s$, and is given by

$$
I(k)=I(0)\left[\operatorname{sinc}\left(\pi s k^{\star}\right)\right]^{2}
$$

for all $k \in L^{\circledast}$. Now, consider a sequence of positions $\tau^{-\ell} k$ with $k \in L^{\circledast}$ and $\ell \in \mathbb{N}_{0}$. Since we have

$$
\operatorname{sinc}(x)=\sin (x) / x=\mathcal{O}\left(x^{-1}\right)
$$

as $x \rightarrow \infty$, it follows that $I\left(\tau^{-\ell} k\right)=\mathcal{O}\left(\tau^{-2 \ell}\right)$ as $\ell \rightarrow \infty$.

Consequently, the sum of intensities along the series of peaks,

$$
\Sigma(k)=\sum_{\ell=0}^{\infty} I\left(\tau^{-\ell} k\right),
$$

satisfies the asymptotic behaviour

$$
\Sigma\left(\tau^{-\ell} k\right) \sim c(k) \tau^{-2 \ell} \Sigma(k)
$$

as $\ell \rightarrow \infty$, where it can be shown that $c(k)=\mathcal{O}(1)$ (Baake $\&$ Grimm, 2019a). Expressing $Z(k)$ in terms of these sums gives

$$
Z(k)=\sum_{\substack{\kappa \in L^{\circledast} \\ \frac{k}{\tau}<\kappa \leq k}} \Sigma(\kappa),
$$

which implies the asymptotic behaviour

$$
Z\left(\tau^{-\ell} k\right) \asymp \tau^{-2 \ell} Z(k) .
$$

This leads to a power-law scaling behaviour of the form $Z(k)=\mathcal{O}\left(k^{2}\right)$ as $k \rightarrow 0^{+}$.

This generic result remains true if we choose a window which corresponds to a tiling with inflation symmetry, which requires the window to be an interval of length $s \in \mathbb{Z}[\tau]$. This obviously holds for our original Fibonacci window $W$ of length $\tau$. However, one gets a stronger result for this case (Baake \& Grimm, 2019a; Oğuz et al., 2017), as we shall now recall.

Choosing $s \in \mathbb{Z}[\tau]$ means $s=a+b \tau$ with $a, b \in \mathbb{Z}$. For $0 \neq k \in L^{\circledast}$, set $k=\kappa / \sqrt{5}$ with $\kappa=m+n \tau$ for some $m, n \in \mathbb{Z}$, excluding $m=n=0$. Applying the $\star$-map then gives

$$
I\left(\tau^{-\ell} k\right)=I(0)\left[\operatorname{sinc}\left(\frac{\pi \tau^{\ell} s \kappa^{\star}}{\sqrt{5}}\right)\right]^{2},
$$

with $\ell \in \mathbb{N}_{0}$.

Now, denote by $f_{n}$ with $n \in \mathbb{Z}$ the Fibonacci numbers defined by $f_{0}=0, f_{1}=1$ and the recursion $f_{n+1}=f_{n}+f_{n-1}$. They satisfy the well-known formula

$$
f_{n}=\frac{1}{\sqrt{5}}\left[\tau^{n}-(-1 / \tau)^{n}\right]
$$

for all $n \in \mathbb{Z}$. Using this relation, we obtain

$$
\begin{aligned}
\sin \left(\frac{\pi \tau^{\ell} s \kappa^{\star}}{\sqrt{5}}\right)^{2} & =\sin \left(\frac{\pi\left|s \kappa^{\star}\right|}{\sqrt{5}} \tau^{-\ell}\right)^{2} \\
& =\frac{\pi^{2}\left(s \kappa^{\star}\right)^{2}}{5} \tau^{-2 \ell}+\mathcal{O}\left(\tau^{-6 \ell}\right)
\end{aligned}
$$

as $\ell \rightarrow \infty$. Here, the first step follows by using equation (29) to replace $\tau^{\ell} / \sqrt{5}$ and then reducing the argument via the relation

$$
\sin (m \pi+x)=(-1)^{m} \sin (x),
$$

which holds for all $m \in \mathbb{Z}$ and $x \in \mathbb{R}$. This is possible because all Fibonacci numbers are integers. The second step then uses the Taylor approximation $\sin (x)=x+\mathcal{O}\left(x^{3}\right)$ for small values of $x$.

Now, the same argument as above implies the asymptotic behaviour 

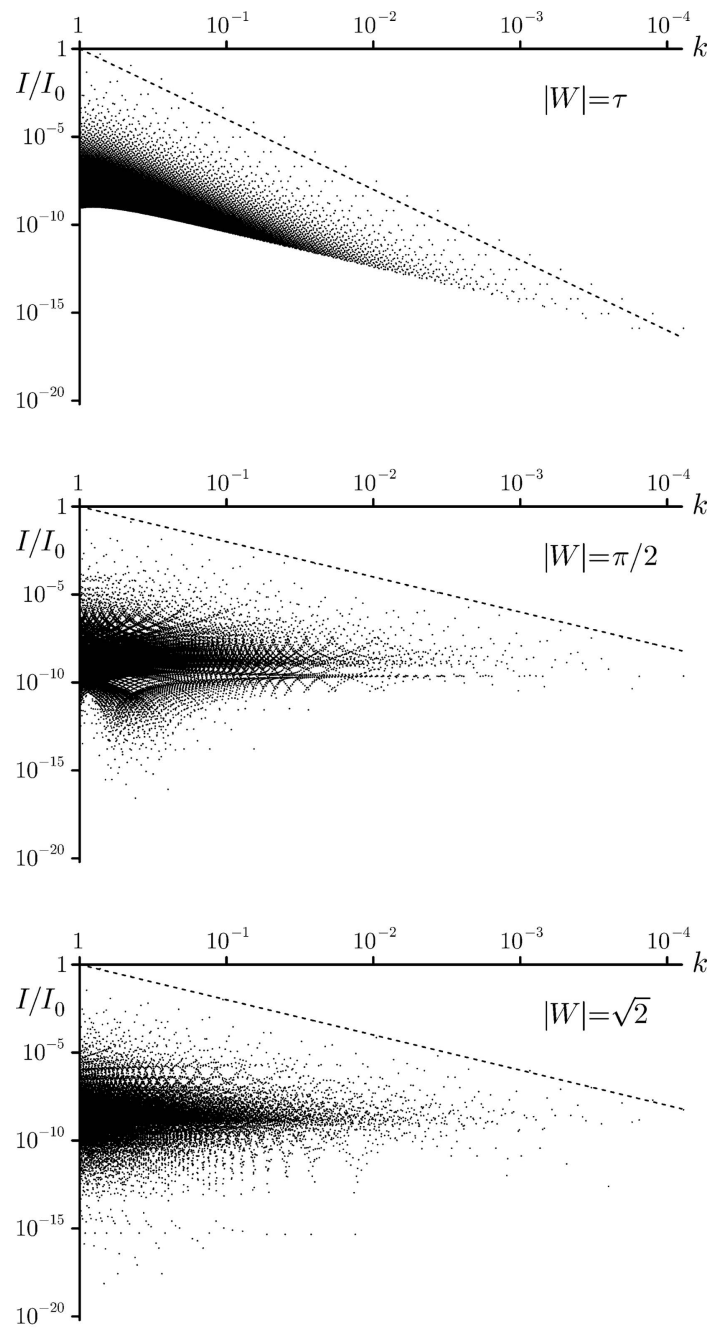

Figure 12

Double logarithmic plot of the intensity ratio $I / I_{0}$ of Bragg peaks located at $k=(m+n \tau) / \sqrt{5}$ with $\max (|m|,|n|) \leq 10^{4}$, where $I_{0}=I(0)$, for windows $W$ of different lengths. The dashed line corresponds to $k^{4}$ for length $|W|=\tau$ (top) and to $k^{2}$ for the other two cases.

$$
Z\left(\tau^{-\ell} k\right) \asymp \tau^{-4 \ell} Z(k),
$$

and hence $Z(k)=\mathcal{O}\left(k^{4}\right)$. This result means that, for inflationinvariant projection sets, the distribution function $Z(k)$ of the diffraction intensity vanishes like $k^{4}$ as $k \rightarrow 0^{+}$, while, in the generic case, we find a $k^{2}$-behaviour. This example illustrates that the behaviour of the diffraction intensity near 0 can pick up non-trivial aspects of order in this system. This is illustrated for some cases in Fig. 12.

Our discussion above may appear quite special, in the sense that we chose all scattering strengths to be equal. However, since we are only interested in the scaling behaviour near the origin, this is in fact no restriction, because the scaling law is unaffected by changing the scattering strengths (as the length of the total window falls into $\mathbb{Z}[\tau]$ if and only if the lengths of the sub-windows do). This simultaneously points to a strength and a weakness of this quantity as a measure of order. On the one hand, the scaling behaviour can detect and distinguish the order in the spatial arrangement of atoms irrespective of the scattering strengths of the atoms; on the other hand, it cannot provide any information on the distribution of different scatterers. For the latter, the knowledge of the intensities of the Bragg peaks is required.

Let us briefly comment on the scaling behaviour for other prominent examples of aperiodic order discussed by Baake \& Grimm (2019a). For noble means inflations, we observe the same $k^{4}$-scaling as for the Fibonacci tiling. The period doubling sequence, which is limit periodic, shows $k^{2}$-scaling, and a range of scaling exponents is accessible for substitutions of more than two letters. For the Thue-Morse sequence, which is the paradigm of an inflation structure with singular continuous diffraction, we do not obtain a power law, but an exponential scaling behaviour which decays faster than any power; see also Baake, Gohlke et al. (2019) for more on the scaling of the spectrum for this system. Finally, the RudinShapiro sequence, which has an absolutely continuous spectrum, shows a linear scaling behaviour, due to the constant density of its diffraction measure.

\section{Acknowledgements}

It is our pleasure to thank Claudia Alfes-Neumann, Natalie Priebe Frank, Neil Mañibo, Bernd Sing, Nicolae Strungaru and Venta Terauds for valuable discussions, and two anonymous referees for useful comments and suggestions.

\section{Funding information}

This work was supported by the German Research Foundation (DFG), within the CRC 1283 at Bielefeld University, and by the EPSRC, through grant EP/S010335/1.

\section{References}

Akiyama, S. \& Arnoux, P. (2020). Editors. Tiling Dynamical Systems: Substitutions and Beyond. Berlin: Springer.

Akiyama, S., Barge, M., Berthé, V., Lee, J.-Y. \& Siegel, A. (2015). Mathematics of Aperiodic Order, edited by J. Kellendonk, D. Lenz \& J. Savinien, pp. 33-72. Basel: Birkhäuser.

Allouche, J.-P. \& Shallit, J. (2003). Automatic Sequences. Cambridge University Press.

Aubry, S., Godrèche, C. \& Luck, J. M. (1988). J. Stat. Phys. 51, $1033-$ 1075.

Baake, M., Coons, M. \& Mañibo, N. (2020). From Analysis to Visualization: JBCC 2017, edited by D. Bailey, N. S. Borwein, R. P. Brent, R. S. Burachik, J.-A. H. Osborn, B. Sims \& Q. J. Zhu, pp. 303-322. Cham: Springer.

Baake, M., Ecija, D. \& Grimm, U. (2016). Z. Kristallogr. 231, 507515.

Baake, M., Frank, N. P. \& Grimm, U. (2021). Stoch. Dyn. 21, 214001.

Baake, M., Frank, N. P., Grimm, U. \& Robinson, E. A. (2019). Stud. Math. 247, 109-154.

Baake, M. \& Gähler, F. (2016). Topol. Appl. 205, 4-27.

Baake, M., Gähler, F. \& Grimm, U. (2013). J. Integer Seq. 16, 13.2.14. Baake, M., Gähler, F. \& Mañibo, N. (2019). Commun. Math. Phys. 370, 591-635.

Baake, M., Gohlke, P., Kesseböhmer, M. \& Schindler, T. (2019). Discrete Cont. Dyn. Syst. A, 39, 4157-4185.

Baake, M. \& Grimm, U. (2003). Discrete Comput. Geom. 30, 573-589.

Baake, M. \& Grimm, U. (2009). Phys. Rev. B, 79, 020203.

Baake, M. \& Grimm, U. (2012). Chem. Soc. Rev. 41, 6821-6843. 
Baake, M. \& Grimm, U. (2013). Aperiodic Order, Vol. 1, A Mathematical Invitation. Cambridge University Press.

Baake, M. \& Grimm, U. (2019a). J. Stat. Mech. 2019, 054003.

Baake, M. \& Grimm, U. (2019b). arXiv:1907.11012.

Baake, M. \& Grimm, U. (2020). J. Phys. Conf. Ser. 1458, 012006.

Baake, M., Grimm, U. \& Mañibo, N. (2018). Lett. Math. Phys. 108, 1783-1805.

Baake, M., Huck, C. \& Strungaru, N. (2017). Indag. Math. 28, 3-31.

Baake, M. \& Zeiner, P. (2017). Aperiodic Order. Vol. 2, Crystallography and Almost Periodicity, edited by M. Baake \& U. Grimm, pp. 73-172. Cambridge University Press.

Bartlett, A. (2018). Ergod. Th. Dyn. Syst. 38, 1289-1341.

Berlinkov, A. \& Solomyak, B. (2019). Ergod. Th. Dyn. Syst. 39, 2384 2402.

Brauchart, J. S., Grabner, P. J. \& Kusner, W. (2019). Constr. Approx, 50, 45-61.

Brauchart, J. S., Grabner, P. J., Kusner, W. B. \& Ziefle, J. (2018). arXiv:1809.02645.

Bufetov, A. \& Solomyak, B. (2018). arXiv:1802.04783.

Bufetov, A. \& Solomyak, B. (2020). arXiv:2003.11287.

Chan, L. \& Grimm, U. (2017). Adv. Appl. Math. 87, 16-23.

Chan, L., Grimm, U. \& Short, I. (2018). Indag. Math. 29, 1072-1086.

Clark, A. \& Sadun, L. (2006). Ergod. Th. Dyn. Syst. 26, 69-86.

Coates, S., Smerdon, J. A., McGrath, R. \& Sharma, H. R. (2018). Nat. Commun. 9, 3435.

Damanik, D. \& Gorodetski, A. (2018). J. Spectr. Theory, 8, 14871507.

Even-Dar Mandel, S. \& Lifshitz, R. (2008). Philos. Mag. 88, 22612273.

Frank, N. P. (2003). Ergod. Th. Dyn. Syst. 23, 519-532.

Frank, N. P. (2015). Mathematics of Aperiodic Order, edited by J. Kellendonk, D. Lenz \& J. Savinien, pp. 223-257. Basel: Birkhäuser.

Frettlöh, D. (2017). Aperiodic Order, Vol. 2, Crystallography and Almost Periodicity, edited by M. Baake \& U. Grimm, pp. 1-37. Cambridge University Press.

Fuchs, J.-N., Mosseri, R. \& Vidal, J. (2019). Phys. Rev. B, 100, 125118. Godrèche, C. \& Luck, J. M. (1990). J. Phys. A Math. Gen. 23, 37693797.

Grimm, U. (2015). Acta Cryst. B71, 258-274.

Hof, A. (1995). Commun. Math. Phys. 169, 25-43.
Kellendonk, J., Lenz, D. \& Savinien, J. (2015). Editors. Mathematics of Aperiodic Order. Basel: Birkhäuser.

Lifshitz, R. (2002). J. Alloys Compd. 342, 186-190.

Luck, J. M. (1993). Europhys. Lett. 24, 359-364.

Mañibo, C. N. (2019). Lyapunov Exponents in the Spectral Theory of Primitive Inflation Systems. PhD thesis, Bielefeld University, Germany. urn:nbn:de:0070-pub-29359727.

Mañibo, N. (2017). J. Math. Phys. 58, 113504.

Moody, R. V. (2000). From Quasicrystals to More Complex Systems, edited by F. Axel, F. Dénoyer \& J. P. Gazeau, pp. 145-166. Berlin: Springer.

Moody, R. V. (2002). Can. Math. Bull. 45, 123-130.

Oğuz, E. C., Socolar, J. E. S., Steinhardt, P. J. \& Torquato, S. (2017). Phys. Rev. B, 95, 054119.

Oğuz, E. C., Socolar, J. E. S., Steinhardt, P. J. \& Torquato, S. (2019). Acta Cryst. A75, 3-13.

Pytheas Fogg, N. (2002). Substitutions in Dynamics, Arithmetics and Combinatorics, LNM 1794. Berlin: Springer.

Queffélec, M. (2010). Substitution Dynamical Systems - Spectral Analysis, 2nd ed., LNM 1294. Berlin: Springer.

Richard, C. \& Strungaru, N. (2017a). J. Phys. A: Math. Theor. 50, 154003.

Richard, C. \& Strungaru, N. (2017b). Ann. Henri Poincaré, 18, 39033931.

Sadun, L. (2008). Topology of Tiling Spaces. Providence: American Mathematical Society.

Schlottmann, M. (2000). Directions in Mathematical Quasicrystals, edited by M. Baake \& R. V. Moody, pp. 143-159. Providence: American Mathematical Society.

Shechtman, D., Blech, I., Gratias, D. \& Cahn, J. W. (1984). Phys. Rev. Lett. 53, 1951-1953.

Solomyak, B. (1997). Ergod. Th. Dyn. Syst. 17, 695-738.

Strungaru, N. (2013). Can. J. Math. 65, 675-701.

Strungaru, N. (2017). Aperiodic Order, Vol. 2, Crystallography and Almost Periodicity, edited by M. Baake \& U. Grimm, pp. 271-342. Cambridge University Press.

Strungaru, N. (2020). J. Funct. Anal. 278, 108404.

Takakura, H., Gómez, C. P., Yamamoto, A., de Boissieu, M. \& Tsai, A. P. (2007). Nat. Mater. 6, 53-63.

Torquato, S. \& Stillinger, F. H. (2003). Phys. Rev. E, 68, 041113. 